BULL. AUSTRAL. MATH. SOC.

VOL. 16 (1977), 161-180.

\title{
Orthodox bands of modules
}

\section{F. Pastijn}

In this paper we shall consider orthodox bands of commutative groups, together with a ring of endomorphisms. We shall generalize the concept of a left module by introducing orthodox bands of left modules; we shall also deal with linear mappings, the transpose of a linear mapping and with the dual of an orthodox band of left modules.

We shall use the notations and terminology of [1] and [3].

1 .

DEFINITION. Let $R,+$, 0 be a ring with zero element 0 and identity 1 . Let $S$ be a semigroup and $R \times S \rightarrow S,(\alpha, x) \mapsto \alpha x$ a mapping satisfying the following conditions:

$$
\begin{aligned}
& \text { (i) } \alpha(x y)=(\alpha x)(\alpha y) \text { for every } \alpha \in R \text { and every } x, y \in S \text {, } \\
& \text { (ii) }(\alpha+\beta) x=(\alpha x)(\beta x) \text { for every } \alpha, \beta \in R \text { and every } x \in S \text {, } \\
& \text { (iii) }(\alpha \circ \beta) x=\alpha(\beta x) \text { for every } \alpha, \beta \in R \text { and every } x \in S \text {, } \\
& \text { (iv) } 1 x=x \text { for every } x \in S .
\end{aligned}
$$

The structure defined this way will be called an orthodox band of left $R$-modules. The next theorem justifies our terminology.

2 .

THEOREM 1. Let $R, S$ and the mapping $R \times S \rightarrow S$ be as in the definition of Section 1. Then $S$ is an orthodox band of abelian groups and the maximal subgroups of $S$ are left invariant by the elements of $R$.

Received 12 October 1976. 
Proof. Let $x$ be any element of $S$, and $\alpha$ any element of $R$; we then have

$$
\begin{aligned}
(0 x)(0 x) & =(0+0) x=0 x, \\
(\alpha x)(0 x) & =(\alpha+0) x=\alpha x=(0+\alpha) x=(0 x)(\alpha x), \\
(\alpha x)((-\alpha) x) & =(\alpha-\alpha) x=0 x=(-\alpha+\alpha) x=((-\alpha) x)(\alpha x) .
\end{aligned}
$$

This implies that for any $\alpha \in R$ and any $x \in S, \alpha x$ belongs to the maximal subgroup of $S$ with identity $0 x$; the inverse of $\alpha x$ in this maximal subgroup must be $(-\alpha) x$. More specifically $l x=x$ belongs to the maximal subgroup of $S$ with identity $0 x$, and its inverse in this maximal subgroup must be $(-1) x$. We conclude that $S$ must be a completely regular semigroup and that all maximal subgroups of $S$ are left invariant by the elements of $R$.

For every $x, y \in S$ we have

$$
(x y)(x y)=(1+1)(x y)=((1+1) x)((1+1) y)=x^{2} y^{2} \text {. }
$$

Let $e, f$ be any idempotents of $S$, then the foregoing implies that $(e f)^{2}=e^{2} f^{2}=e f$; hence $E_{S}=\left\{x \in S \mid x^{2}=x\right\}$ must be a subsemigroup of $S$. Let $x$ and $y$ belong to the same maximal subgroup of $S$; then the foregoing implies

$$
x y=((-1) x) x^{2} y^{2}((-1) y)=((-1) x) x y x y((-1) y)=y x ;
$$

hence $S$ is a union of abelian groups. We have yet to prove that $S$ is an orthodox union of abelian groups [2].

Let $e$ and $f$ be any idempotents of $S$, and $x \in H_{e}, y \in H_{f}$. We put $(-1) x=x^{\prime}$ and $(-1) y=y^{\prime}$. Then

$$
e f=(e f)^{2}=(1+1)(e f)=(1+1)\left(x\left(x^{\prime} f\right)\right)=x^{2}\left(x^{\prime} f\right)^{2}=x^{2} x^{\prime} f x^{\prime} f=(x f)\left(x^{\prime} f\right)
$$

and analogously

$$
e f=\left(x^{\prime} f\right)(x f) \text {. }
$$

Since ef, $x^{\prime} f$, and $x f$ are elements of the rectangular group $D_{\text {ef }}[2]$, the foregoing implies that $x f$ and $x^{\prime} f$ are mutually inverse elements of the maximal subgroup $H_{e f}$ ' Dually, $e y$ and $e y$ ' are mutually inverse 
elements of the maximal subgroup $H_{e f}$. Since $(x y) y^{\prime}=x f$ and $(x f) y=x y$ we have $x y R x f$; hence $x y R e f$. Analogously, since $x^{\prime}(x y)=e y$ and $x(e y)=x y$ we have $x y$ Ley; hence $x y$ Lef. We conclude that xyHef. Green's relation $H$ must then be a congruence on $S$. Thus $S$ is an orthodox band of commutative groups [2].

3.

REMARK. Let $S$ be an orthodox band of abelian groups. Then, by Yamada's Theorem ([2] and [10]), there exists a band $E$ and a semilattice of abelian groups $Q$, both having the same structure semilattice $Y$, such that $S$ is the spined product of $Q$ and $E$ over $Y: S=Q \times{ }_{Y} E$. Let $Q=\underset{K \in Y}{U} G_{K}$ and $E=\underset{K \in Y}{U} E_{K} ;$ then $S$ consists of ordered pairs $\left(x_{K}, e_{K}\right), K \in Y, x_{K} \in G_{K}, e_{K} \in E_{K}$. Multiplication is defined by

$$
\left(x_{\lambda}, e_{\lambda}\right)\left(y_{\mu}, f_{\mu}\right)=\left(x_{\lambda} y_{\mu}, e_{\lambda} f_{\mu}\right)
$$

for any $\lambda, \mu \in Y, x_{\lambda} \in G_{\lambda}, y_{\mu} \in G_{\mu}, e_{\lambda} \in E_{\lambda}, f_{\mu} \in E_{\mu}$. The identity element of $G_{\kappa}, \kappa \in Y$, will be denoted by $I_{\kappa}$.

The following result will generalize a theorem of [4] about semilattices of left modules. By combining the next theorem and Theorem 1 , we obtain a characterization of orthodox bands of abelian groups.

4.

THEOREM 2. Let $S$ be any orthodox band of abelian groups, and let $\mathrm{Z}$ be the ring of integers. Let $e$ be any idempotent of $S$, and $x$ and $x^{\prime}$ mutually inverse elements of the maximal subgroup $H_{e}$. Define the mapping $\mathrm{Z} \times S \rightarrow S, \quad(k, x) \mapsto k x$ by

$$
\begin{array}{rlrl}
k x & =x^{k} & & \text { if } k>0 \\
& =e & & \text { if } k=0 \\
& =x^{1^{-k}} & \text { if } k<0 .
\end{array}
$$

Then $S$ is an orthodox band of left Z-modules. 
Proof. Conditions (i), (ii), (iii), and (iv) of the definition in Section 1 are checked by some easy calculations.

5.

DEFINITIONS and REMARKS. Let $S$ be an orthodox band of left $R$-modules, and $\tau$ a congruence on the semigroup $S$. The natural homomorphism of $S$ onto $S / \tau$ will be denoted by $\tau^{\#}$. $\tau$ will be called $R$-stable if and only if $x \tau y$ implies $(\alpha x) \tau(\alpha y)$ for every $x, y \in S$ and every $\alpha \in R$; we can then define a mapping $R \times(S / \tau) \rightarrow S / \tau$ by $(\alpha, \bar{x}) \mapsto \alpha \bar{x}=\overline{\alpha x} ; S / \tau$ will then be an orthodox band of left $R$-modules.

Let $S$ and $T$ be orthodox bands of left $R$-modules. The mapping $\Phi: S \rightarrow T$ will be called $R$-linear if and only if

(i) $\Phi(x y)=(\Phi x)(\Phi y)$ for every $x, y \in S$,

(ii) $\Phi(\alpha x)=\alpha \Phi(x)$ for every $x \in S$ and every $\alpha \in R$.

$\Phi(S)$ will then be an orthodox band of left $R$-modules.

The subset $A$ of $S$ will be called $R$-stable if and only if $\alpha x \in A$ for every $x \in A$ and every $\alpha \in R$. If $\Phi$ is an $R$-linear mapping of $S$ into $T, \Phi(S)$ will be an R-stable subsemigroup of $T$, and the kernel of $\Phi$ will be an $R$-stable subsemigroup of $S$. Any $R$-stable subsemigroup of an orthodox band of left $R$-modules must of course be an orthodox band of left $R$-modules. If $\tau$ is an $R$-stable congruence on $S$, the union of all $\tau$-classes containing an idempotent will be an $R$-stable subsemigroup of $S$.

The mapping $\Phi: S \rightarrow T$ will be $R$-linear if and only if $\Phi^{-1} \Phi$ is an $R$-stable congruence on $S$. The equivalence relation $\tau$ on $S$ is an $R$-stable congruence if and only if $\tau^{\#}$ is an $R$-linear mapping. The mapping $\Phi: S \rightarrow E_{S}, x \mapsto 0 x$ is an $R$-linear mapping of $S$ onto the band consisting of all idempotents of $S ; \Phi^{-1} \Phi$ is then the $R$-stable congruence $H$.

Let $S$ be the spined product of a semilattice of abelian groups $Q$ and a band $E$; we shall use the same notation as in the remark of Section 3. $Q$ is the greatest inverse semigroup homomorphic image of $S$, and the 
mapping $\Delta: S \rightarrow Q,\left(x_{k}, e_{k}\right) \mapsto x_{k}$ is a homomorphism of $S$ onto $Q$; we shall put $\Delta^{-1} \Delta=\sigma$; this congruence $\sigma$ is the minimal inverse semigroup congruence on $S$, and we shall show that $\sigma$ is $R$-stable. Let $G$ be the greatest group homomorphic image of $Q$, and $\Gamma: Q \rightarrow G, x_{K} \mapsto \tilde{x}_{K}$ be a homomorphism of $Q$ onto $G, \Gamma^{-1} \Gamma$ being the minimal group congruence on $Q$; if $x_{\lambda}$ and $y_{\mu}$ are any elements of $Q$, then $x_{\lambda} \Gamma^{-1} \Gamma y_{\mu}$ if and only if there exists a $\kappa \in Y, \quad \leqslant \leq \lambda \wedge \mu$, such that $x_{\lambda^{1}{ }_{K}}=y_{\mu^{1} \kappa}$; we shall put $(\Gamma \Delta)^{-1}(\Gamma \Delta)=\rho$; this congruence $\rho$ is the minimal group congruence on $S$, and we shall show that $\rho$ is $R$-stable.

\section{6.}

THEOREM 3. The minimal inverse semigroup congruence on an orthodox band of left R-modules is R-stable.

Proof. Let $x_{k}$ be any element of $Q$, and let us take any two elements $\left(x_{K}, e_{K}\right)$ and $\left(x_{K}, f_{K}\right)$ in $\Delta^{-1} x_{K}$. Let $\alpha$ be any element of $R$. Since $H$ is an $R$-stable congruence on $S, \alpha\left(x_{K}, e_{K}\right)$ belongs to the H-class $G_{K} \times e_{K}$ of $S$ containing $\left(x_{K}, e_{K}\right)$; hence $\alpha\left(x_{K}, e_{K}\right)=\left(y_{K}, e_{K}\right)$ for some $y_{K} \in G_{K}$. Analogously, $\alpha\left(x_{K}, f_{K}\right)=\left(z_{K}, f_{K}\right)$ for some $z_{K} \in G_{K}$. Let $\left(I_{K}, g_{K}\right)$ be L-related with $\left(I_{K}, e_{K}\right)$ and R-related with $\left(I_{K}, f_{K}\right)$, and let $\left(1_{K}, h_{K}\right)$ be R-related with $\left(I_{K}, e_{K}\right)$ and L-related with $\left(I_{K}, f_{K}\right)$. Since by the restriction of $R \times S+S$ to $R \times\left(G_{\mathrm{K}} \times g_{\mathrm{K}}\right)$ and $R \times\left(G_{\mathrm{K}} \times h_{\mathrm{K}}\right)$, respectively, $G_{K} \times g_{K}$ and $G_{K} \times h_{K}$ become left $R$-modules, we must have $\alpha\left(I_{K}, g_{K}\right)=\left(I_{K}, g_{K}\right)$ and $\alpha\left(I_{K}, h_{K}\right)=\left(I_{K}, h_{K}\right)$. Furthermore, we have

$$
\begin{aligned}
\left(z_{K}, e_{K}\right) & =\left(1_{K}, h_{K}\right)\left(z_{K}, f_{K}\right)\left(1_{K}, g_{K}\right) \\
& =\left(\alpha\left(1_{K}, h_{K}\right)\right)\left(\alpha\left(x_{K}, f_{K}\right)\right)\left(\alpha\left(1_{K}, g_{K}\right)\right) \\
& =\alpha\left(\left(1_{K}, h_{K}\right)\left(x_{K}, f_{K}\right)\left(1_{K}, g_{K}\right)\right) \\
& =\alpha\left(x_{K}, e_{K}\right)=\left(y_{K}, e_{K}\right) ;
\end{aligned}
$$


hence $z_{K}=y_{K}$, and $\Delta\left(\alpha\left(x_{K}, e_{K}\right)\right)=\Delta\left(\alpha\left(x_{K}, f_{K}\right)\right)$.

7.

COROLLARY 1. By the mapping $R \times Q \rightarrow Q$,

$$
\left(\alpha, x_{\kappa}\right) \mapsto \alpha x_{K}=\Delta\left(\alpha \Delta-\frac{1}{x_{K}}\right) \text {, }
$$

$Q$ becomes a semilattice of left R-modules, and $\Delta$ an $R$-linear mapping of $S$ onto $Q$.

8.

COROLLARY 2. Let $Q$ be any semilattice of left R-modules, and $Y$ the structure semilattice of $Q$; let $E$ be a band with the same structure semilatice $Y$; let $\underset{K \in Y}{U} G_{K}$ and $\underset{K \in Y}{U} E_{K}$ be the semilatice decompositions of $Q$ and $E$ respectively; let $S$ be the spined product $Q \times{ }_{Y} E$ of $Q$ and $E$ over $Y$. By the mapping $R \times S \rightarrow S$, $\left(\alpha,\left(x_{\kappa}, e_{K}\right)\right) \mapsto\left(\alpha x_{\kappa}, e_{K}\right)$ for every $\alpha \in R$, and every $\kappa \in Y$, $x_{K} \in G_{K}, e_{K} \in E_{K}, S$ becomes an orthodox band of left R-modules. Conversely, any orthodox band of left $R$-modules can be so constructed.

9.

COROLLARY 3. Let $S$ be an orthodox normal band of left R-modules, and let $S=\bigcup_{K \in Y} S_{K}$ be the semilattice decomposition of $S$. For any $\lambda$, $\mu \in Y, \lambda \geq \mu$, the structure homomorphism $\Psi_{\lambda, \mu}$ is an R-linear mapping of the orthodox rectangular band of left R-modules $S_{\lambda}$ into the orthodox rectangular band of left $R$-modules $S_{\mu}$.

Proof. In a semilattice of left $R$-modules the structure homomorphisms are $R$-linear [6]. The theorem now follows from Corollary 2 and from a result about normal bands [11].

10.

REMARK. Structure theorems for semilattices of left $R$-modules [6], 
together with Corollary 2 yield structure theorems for bands of left $R$-modules.

11.

THEOREM 4. The minimal group congruence on an orthodox band of left R-modules is R-stable.

Proof. Let $\tilde{x}_{\lambda}$ be any element of $G$, the greatest group homomorphic image of an orthodox band of left $R$-modules $S$. Let us take any two elements $x_{\lambda}$ and $y_{\mu}$ in $\Gamma^{-1} \tilde{x}_{\lambda}$. There exists a $\kappa \in Y, \kappa \leq \lambda \wedge \mu$, such that $1_{\kappa} x_{\lambda}=1_{\kappa} y_{\mu}$. Let $\alpha$ be any element of $R$. From

$$
\left(\alpha x_{\lambda}\right) 1_{K}=\left(\alpha x_{\lambda}\right)\left(\alpha l_{K}\right)=\alpha\left(x_{\lambda^{1}{ }_{K}}\right)=\alpha\left(y_{\mu^{1}{ }_{K}}\right)=\left(\alpha y_{\mu}\right)\left(\alpha I_{K}\right)=\left(\alpha y_{\mu}\right) I_{K} \text {, }
$$

and $\alpha x_{\lambda} \in G_{\lambda}, \alpha y_{\mu} \in G_{\mu}$, we conclude that $\alpha y_{\mu} \in \Gamma^{-1} \Gamma\left(\alpha x_{\lambda}\right)$, and thus $\tilde{\alpha x}_{\lambda}=\tilde{\alpha y}_{\mu}$. This implies that the minimal group congruence $\Gamma^{-1} \Gamma$ on $Q$ must be $R$-stable; consequently, the minimal group congruence $(\Gamma \Delta)^{-1} \Gamma \Delta=\rho$ on $S$ must be $R$-stable.

12 .

COROLLARY 4. By the mapping $R \times G \rightarrow G, \quad\left(\alpha, \tilde{x}_{K}\right) \mapsto \alpha \tilde{x}_{K}=\tilde{\alpha x}_{K}$, $G$ becomes a left $R$-module, and the mapping $\Gamma \Delta$ an R-linear mapping of $S$ onto $G$.

13.

DEFINITIONS. An orthodox band of right $R$-modules $S$ can be defined in a way analogous to the way an orthodox band of left $R$-modules is defined. Condition (iii) of the definition in section 1 must then be repleced by (iii)'; $(\alpha \circ \beta) x=\beta(\alpha x)$ for every $\alpha, \beta \in R$ and every $x \in S$. It will be more convenient to denote the mapping $R \times S \rightarrow S$ by $(\alpha, x) \mapsto x \alpha ;(i i i)$ ' then becomes

$$
\text { (iii)' } x(\alpha \circ \beta)=(x \alpha) \beta \text { for every } \alpha, \beta \in R \text { and every } x \in S \text {. }
$$

If $S$ is at the same time an orthodox band of left $R$-modules, and an 
orthodox band of right $R$-modules, then we shall say that $S$ is an orthodox band of $R$-bimodules.

Let $R^{\infty}=R \cup\{\infty\}$, and define addition in $R^{\infty}$ as follows: for any $\alpha, \beta \in R$ we put

$$
\alpha+\beta=\gamma \text { in } R^{\infty} \text { if and only if } \alpha+\beta=\gamma \text { in } R \text {, }
$$

and we put

$$
\alpha+\infty=\infty+\alpha=\infty \text {. }
$$

$R^{\infty}$ will be a group with "zero" $\infty$. We next define the mapping $R \times R^{\infty} \rightarrow R^{\infty}$ by agreeing that for $\alpha, \beta$ in $R$,

$$
(\alpha, \beta) \mapsto \alpha \beta=\gamma \text { if and only if } \alpha \circ \beta=\gamma \text { in } R \text {, }
$$

and that

$$
(\alpha, \infty) \mapsto \alpha_{\infty}^{\infty}=\infty \text {. }
$$

We also define the mapping $R \times R^{\infty} \rightarrow R^{\infty}$ by setting, for $\alpha, \beta$ in $R$,

$$
(\alpha, \beta) \mapsto \beta \alpha=\gamma \text { if and only if } \beta \circ \alpha=\gamma \text { in } R \text {, }
$$

and

$$
(\alpha, \infty) \mapsto \infty \alpha=\infty \text {. }
$$

By these two mappings $R^{\infty}$ becomes a semilattice of $R$-bimodules, the structure semilattice being the two element semilattice. We shall use $R^{\infty}$ later in this paper.

The next theorem generalizes a result of [9].

14.

THEOREM 5. Let $S$ be an orthodox band of left R-modules, and $T$ an orthodox band of right R-modules. Let $I_{S, T}$ be the set of all partial mappings of $S$ into $T$. Define a multiplication in $I_{S, T}$ as follows: for every $\Phi, \Psi \in I_{S, T}, \operatorname{dom} \Phi \Psi=\operatorname{dom} \Phi \cap \operatorname{dom} \Psi$, and for every $x \in \operatorname{dom} \Phi \Psi$ we put $\Phi \Psi(x)=(\Phi x)(\Psi x)$. Define the mapping $R \times I_{S, T} \rightarrow I_{S, T}, \quad(\alpha, \Phi) \mapsto \Phi \alpha$ by $\operatorname{dom}(\Phi \alpha)=\operatorname{dom} \Phi$ and $(\Phi \alpha) x=(\Phi x) \alpha$, for every $x \in \operatorname{dom} \Phi . I_{S, T}$ will then be an orthodox band of right R-modules. I $I_{S, T}$ wilz be a semizattice of right $R$-modules if and only if 
$T$ is a semilatice of right $R$-modules.

Proof. For any $\Phi, \Psi \in I_{S, T}$ and any $\alpha \in R$ we have $\operatorname{dom}((\Phi \Psi) \alpha)=\operatorname{dom} \Phi \Psi=\operatorname{dom} \Phi \cap \operatorname{dom} \Psi=\operatorname{dom}(\Phi \alpha) \cap \operatorname{dom}(\Psi \alpha)=\operatorname{dom}((\Phi \alpha)(\Psi \alpha))$, and for any $x \in \operatorname{dom}(\Phi \Psi) \alpha$ we have $((\Phi \Psi) \alpha) x=((\Phi \Psi) x) \alpha=((\Phi x)(\Psi x)) \alpha=((\Phi x) \alpha)((\Phi x) \alpha)=$ $=((\Phi \alpha) x)((\Phi \alpha) x)=\left((\Phi \alpha)\left(\Psi_{\alpha}\right)\right) x ;$

hence $(\Phi \Psi) \alpha=(\Phi \alpha)(\Psi \alpha)$. For any $\Phi \in I_{S, T}$ and any $\alpha, \beta \in R$ we have

$$
\operatorname{dom}(\Phi(\alpha+\beta))=\operatorname{dom} \Phi=\operatorname{dom}(\Phi \alpha) \cap \operatorname{dom}(\Phi \beta)=\operatorname{dom}((\Phi \alpha)(\Phi \beta)),
$$

and for any $x \in \operatorname{dom}(\Phi(\alpha+\beta))$ we have

$$
(\Phi(\alpha+\beta)) x=(\Phi x)(\alpha+\beta)=((\Phi x) \alpha)((\Phi x) \beta)=((\Phi \alpha) x)((\Phi \beta) x)=((\Phi \alpha)(\Phi \beta)) x ;
$$

hence $\Phi(\alpha+\beta)=(\Phi \alpha)(\Phi \beta)$. Furthermore,

$$
\operatorname{dom}(\Phi(\alpha \circ \beta))=\operatorname{dom} \Phi=\operatorname{dom}(\Phi \alpha)=\operatorname{dom}((\Phi \alpha) \beta),
$$

and for any $x \in \operatorname{dom}(\Phi(\alpha \circ \beta))$ we have

$$
(\Phi(\alpha \circ \beta)) x=(\Phi x)(\alpha \circ \beta)=((\Phi x) \alpha) \beta=((\Phi \alpha) x) \beta=((\Phi \alpha) \beta) x ;
$$

hence $\Phi(\alpha \circ \beta)=(\Phi \alpha) \beta$. Finally, $\operatorname{dom}(\Phi 1)=\operatorname{dom} \Phi$, and for any $x \in \operatorname{dom}(\Phi 1)$ we have

$$
(\Phi 1) x=(\Phi x) 1=\Phi x ;
$$

hence $\Phi I=\Phi$. We conclude that $I_{S, T}$ is an orthodox band of right $R$-modules .

From the definition of the multiplication in $I_{S, T}$ it follows that $I_{S, T}$ is commutative if and only if $T$ is commutative. From this follows the last part of the theorem.

15.

THEOREM 6. Let $S$ be an orthodox band of left R-modules, $S$ ' the set of $R$-linear mappings of $S$ into $R$, and $S^{*}$ the set of $R$-linear mappings of $S$ into $R^{\infty}$. Then $S^{\prime}$ is an R-stable subsemigroup of ${ }^{I} S, R$ and $S^{*}$ is an R-stable subsemigroup of $I_{S, R}$. 
Proof. We show that $S^{*}$ is an $R$-stable subsemigroup of ${ }_{S, R^{\infty}}$; the proof of the rest is similar. Let $x^{*}$ and $y^{*}$ be any elements of $S^{*}$; since $R^{\infty}$ is a semilattice of commutative groups, $x^{*} y^{*}$ must be a homomorphism of $S$ into $R^{\infty}$. For any $x \in S$ and any $x^{*} \in S^{*}$ we shall from now on put $x^{*}(x)=\left\langle x, x^{*}\right\rangle$. For any $x \in S$, any $\alpha \in R$, and any $x^{*}, y^{*} \in S^{*}$ we then have

$$
\begin{aligned}
\left\langle\alpha x, x^{*} y^{*}\right\rangle & =\left\langle\alpha x, x^{*}\right\rangle+\left\langle\alpha x, y^{*}\right\rangle \\
& \left.\left.=\alpha x, x^{*}\right\rangle+\alpha x, y^{*}\right\rangle \\
& \left.=\alpha\left(\left\langle x, x^{*}\right\rangle+x, y^{*}\right\rangle\right) \\
& \left.=\alpha x, x^{*} y^{*}\right\rangle .
\end{aligned}
$$

We conclude that for any $x^{*}, y^{*} \in S^{*}, x^{*} y^{*}$ must be an R-linear mapping of $S$ into $R^{\infty}$; hence $x^{*} y^{*} \in S^{*} . S^{*}$ is a subsemigroup of $I_{S, R^{\infty}}$.

For any $x, y \in S$, any $x^{*} \in S^{*}$, and any $\alpha \in R$ we have

$$
\begin{aligned}
\left\langle x y, x^{*} \alpha\right) & =\left(x y, x^{*}\right\rangle \alpha \\
& \left.=\left(\left(x, x^{*}\right\rangle+y, x^{*}\right\rangle\right) \alpha \\
& =\left(x, x^{*}\right) \alpha+\left\langle y, x^{*}\right)_{\alpha} \\
& =\left\langle x, x^{*} \alpha\right\rangle+\left\langle y, x^{*} \alpha\right\rangle ;
\end{aligned}
$$

hence $x^{*} \alpha$ must be a homomorphism of $S$ into $R^{\infty}$. For any $x \in S$, any $x^{*} \in S^{*}$, and any $\alpha, \beta \in R$ we have

$$
\begin{aligned}
\left\langle\beta x, x^{*} \alpha\right) & =\left\langle\beta x, x^{*}\right\rangle \alpha \\
& =\beta\left\langle x, x^{*}\right\rangle \alpha \\
& =\beta\left\langle x, x^{*} \alpha\right\rangle .
\end{aligned}
$$

We conclude that for any $x^{*} \in S^{*}$ and any $\alpha \in R, x^{*} \alpha$ must be an $R$-linear mapping of $S$ into $R^{\infty}$. Consequently $S^{*}$ must be an $R$-stable subsemigroup of $I_{S, R^{\infty}}$.

16.

COROLLARY 5. $S^{*}$ is a semilattice of right $R$-modules. The structure semilattice of $S^{*}$ is isomorphic with the U-semilattice of prime ideals of $S$. The mapping $1^{*}: S \rightarrow R^{\infty}, x \mapsto 0$ is the identity of $S^{*}$ and the mapping $0^{*}: S \rightarrow R^{\infty}, x \mapsto \infty$ is the zero of $S^{*}$. 
Proof. $R^{\infty}$ is a semilattice of right $R$-modules; hence $I_{S, R^{\infty}}$ is a semilattice of right $R$-modules. Since $S^{*}$ is $R$-stable in $I_{S, R^{\infty}}, S^{*}$ must also be a semilattice of right $R$-modules.

Let $e^{*}$ be any idempotent of $S^{*}$; then $V_{e^{*}}=\left\{x \in S \|\left\langle x, e^{*}\right\rangle=\infty\right\}$ is a prime ideal of $S$. For any $x \in S \backslash V_{e^{*}},\left\langle x, e^{*}\right\rangle \in R$ and $\left\langle x, e^{*}\right\rangle=\left\langle x, e^{*^{2}}\right\rangle=\left\langle x, e^{*}\right\rangle+\left\langle x, e^{*}\right\rangle$; hence $\left\langle x, e^{*}\right\rangle=0$. Conversely, let $P$ be any prime ideal of $S$; then we can define $e_{P}^{*} \in S^{*}$ by $\left\langle x, e_{P}^{*}\right\rangle=\infty$ for all $x \in P$, and $\left\langle x, e_{P}^{*}\right\rangle=0$ for all $x \in S \backslash P$. Furthermore, if $e^{*}$ and $f^{*}$ are any two idempotents of $S^{*}$, we must have $V_{e^{*} f^{*}}=V_{e^{*}} \cup V_{f^{*}}$. Consequently, the semilattice $E_{S^{*}}$ consisting of the idempotents of $S^{*}$ is isomorphic with the U-semilattice of all prime ideals of $S$. Since $E_{S^{*}}$ is isomorphic with the structure semilattice of $S^{*}$, the result stated in the corollary follows.

17.

COROLLARY 6. $S^{\prime}$ is a right R-moduze which is an R-stable subgroup of $S^{*} ; S^{\prime}$ is the maximal submodule of $S^{*}$ containing the identity $1^{*}$ of $S^{*}$.

Proof. All elements of $S^{\prime}$ are $R$-linear mappings of $S$ into $R$; hence they can be considered as $R$-linear mappings of $S$ into $R^{\infty}$, and consequently $S^{\prime} \subseteq S^{*}$. Since $S^{\prime}$ is $R$-stable in $I_{S, R}$, and since clearly $I_{S, R}$ is $R$-stable in $I_{S, R^{\infty}}, S^{\prime}$ must be $R$-stable in $I_{S, R^{\infty}}$; from this we infer that $S^{\prime}$ is $R$-stable in $S^{*}$.

It is evident that $1^{*}: S \rightarrow R, x \mapsto 0$ is the identity of $S^{\prime}$. Let $x^{*}$ be any element of $S^{\prime}$; then $x^{*}(-1) \in S^{\prime}$, and for any $x \in S$ we have

$$
\begin{aligned}
\left\{x, x^{*}\left(x^{*}(-1)\right\}\right\rangle & =\left\langle x, x^{*}\right\rangle+\left(x, x^{*}(-1)\right\rangle \\
& =\left\langle x, x^{*}\right\rangle+\left\langle x, x^{*}\right\rangle(-1)=0,
\end{aligned}
$$

and analogously

$$
\left\langle x,\left(x^{*}(-1)\right) x^{*}\right\rangle=0 ;
$$


hence $x^{*}\left(x^{*}(-1)\right)=\left(x^{*}(-1)\right) x^{*}=1^{*}$. This shows that $x^{*}$ and $x^{*}(-1)$ are mutually inverse elements of the commutative group $H_{1^{*}}$, the maximal subgroup of $S^{*}$ containing $1^{*}$. For any element $y^{*} \in H_{1^{*}}$, we must have $V_{y^{*}}=\varnothing$; hence any element $y^{*} \in H_{1 *}$ belongs to $S^{\prime}$. We can conclude that $H_{1 *}=S^{\prime}$.

18.

THEOREM 7. Let $S$ be an orthodox band of left R-modules and $\tau$ any $R$-stable congruence on $S$. The mapping $\Phi:(S / \tau)^{*} \rightarrow S^{*}, \bar{x}^{*} \mapsto \Phi \bar{x}^{*}$ defined by $\left\langle x, \Phi \bar{x}^{*}\right\rangle=\left\langle\tau^{\#} x, \bar{x}^{*}\right\rangle$ for every $x \in S$ is an R-isomorphism of $(S / \tau) *$ into $S^{*}$. Whenever $\iota_{S} \subseteq \tau \subseteq \sigma$, $\sigma$ being the minimal inverse semigroup congruence on $S$, this mapping $\Phi$ is a surjective $R$-isomorphism of $(S / \tau) *$ onto $S^{*}$.

Proof. Let us suppose that $\bar{x}^{*}, \bar{y}^{*}$ are any elements of $(S / \tau)^{*}$, and $x$ any element of $S$; we then have

$$
\begin{aligned}
\left\langle x, \Phi\left(\bar{x}^{*} \bar{y}^{*}\right)\right\rangle & =\left\langle\tau^{\#} x, \bar{x}^{*} \bar{y}^{*}\right\rangle \\
& =\left\langle\tau^{\#} x, \bar{x}^{*}\right\rangle+\left\langle\tau^{\#} x, \bar{y}^{*}\right\rangle \\
& =\left\langle x, \Phi \bar{x}^{*}\right\rangle+\left\langle x, \Phi \bar{y}^{*}\right\rangle \\
& =\left\langle x,\left(\Phi \bar{x}^{*}\right)\left(\Phi \bar{y}^{*}\right)\right\rangle ;
\end{aligned}
$$

hence $\Phi\left(\bar{x}^{*} \bar{y}^{*}\right)=\left(\Phi \bar{x}^{*}\right)\left(\Phi \bar{y}^{*}\right)$. Let us suppose that $\bar{x}^{*}$ is any element of $(S / \tau)^{*}, \alpha$ any element of $R$, and $x$ any element of $S$; then

$$
\begin{aligned}
\left(x, \Phi\left(\bar{x}^{*} \alpha\right)\right\rangle & =\left\langle\tau^{\#} x, \bar{x}^{*} \alpha\right\rangle \\
& =\left\langle\tau^{\#} x, \bar{x}^{*}\right\rangle \alpha \\
& =\left\langle x, \Phi \bar{x}^{*}\right\rangle \alpha \\
& =\left\langle x,\left(\Phi \bar{x}^{*}\right) \alpha\right\rangle ;
\end{aligned}
$$

hence $\Phi\left(\bar{x}^{*} \alpha\right)=\left(\Phi \bar{x}^{*}\right) \alpha$. Since $\tau^{\#}$ is an $R$-linear mapping of $S$ onto $S / \tau, \Phi \bar{x}^{*} \in S^{*}$ for any $\bar{x}^{*} \in(S / \tau)^{*}$. We conclude that $\Phi$ is an $R$-linear mapping of $(S / \tau)^{*}$ into $S^{*}$. Let us now suppose that $\bar{x}^{*}, \bar{y}^{*} \in(S / \tau)^{*}$, and $\Phi \bar{x}^{*}=\Phi_{\bar{y}}^{*} ;$ if for some $\bar{x} \in S / \tau$, $\left\langle\bar{x}, \bar{x}^{*}\right\rangle \neq\left(\bar{x}, \bar{y}^{*}\right)$, then for any $x \in\left(\tau^{\#}\right)^{-1} \bar{x}$ we should have 


$$
\begin{aligned}
\left\langle x, \Phi \bar{x}^{*}\right\rangle & =\left\langle\tau^{\#} x, \bar{x}^{*}\right\rangle \\
& =\left\langle\bar{x}, \bar{x}^{*}\right\rangle \\
& \neq\left\langle\bar{x}, \bar{y}^{*}\right\rangle=\left\langle\tau^{\#} x, \bar{y}^{*}\right\rangle=\left\langle x, \Phi \bar{y}^{*}\right\rangle,
\end{aligned}
$$

and this is impossible. We conclude that $\Phi \bar{x}^{*}=\Phi \bar{y}^{*}$ implies $\bar{x}^{*}=\bar{y}^{*}$; hence $\Phi$ is an isomorphism of $(S / \tau) *$ into $S^{*}$.

It will be sufficient to show that the mapping $\Phi:(S / \sigma)^{*} \rightarrow S^{*}$, $\bar{x}^{*} \mapsto \Phi \bar{x}^{*}$ defined by $\left\langle x, \Phi x^{*}\right\rangle=\left\langle\sigma^{\#} x, \bar{x}^{*}\right\rangle$ for every $x \in S$, will be an $R$-isomorphism of $(S / \sigma)^{*}$ onto $S^{*}$. Let $x^{*}$ be any element of $S^{*}$, and $\left(x_{K}, e_{K}\right)$ and $\left(x_{K}, f_{K}\right)$ any two $\sigma$-related elements of $S$. Since $\left(x_{K}, e_{K}\right)$ and $\left(x_{K}, f_{K}\right)$ are D-related in $S$, they generate the same principal ideal of $S$, and thus $\left.\left(x_{K}, e_{K}\right), x^{*}\right\rangle=\infty$ if and only if $\left(\left(x_{K}, f_{K}\right), x^{*}\right)=\infty$. Let us now suppose that $\left(x_{K}, e_{K}\right)$ and $\left(x_{K}, f_{K}\right)$ both belong to $S \backslash V_{x^{*}}$; let $\left(l_{K}, g_{K}\right)$ be L-related with $\left(x_{K}, e_{K}\right)$ and R-related with $\left(I_{K}, f_{K}\right)$, and $\left(I_{K}, h_{K}\right)$ R-related with $\left(x_{K}, e_{K}\right)$ and L-related with $\left(I_{K}, f_{K}\right) ;\left(I_{K}, g_{K}\right)$ and $\left(I_{K}, h_{K}\right)$ are both 0 -related with $\left(x_{K}, e_{K}\right)$ and $\left(x_{K}, f_{K}\right)$; hence $\left(I_{K}, g_{K}\right),\left(I_{K}, h_{K}\right) \in S \backslash V_{x^{*}}$. Since these two elements are idempotents of $S$, and since $x^{*}$ is an homomorphism of $S \backslash V_{x^{*}}$ into $R$, we have

$$
\left\langle\left(1_{K}, g_{K}\right), x^{*}\right\rangle=\left\langle\left(I_{K}, h_{K}\right), x^{*}\right\rangle=0 \text {. }
$$

From this it pollows that

$$
\begin{aligned}
\left.\left(x_{K}, e_{K}\right), x^{*}\right\rangle & =\left(\left(I_{K}, h_{K}\right)\left(x_{K}, f_{K}\right)\left(I_{K}, g_{K}\right), x^{*}\right\rangle \\
& =\left\langle\left(I_{K}, h_{K}\right), x^{*}\right\rangle+\left\langle\left(x_{K}, f_{K}\right), x^{*}\right)+\left\langle\left(I_{K}, g_{K}\right), x^{*}\right\rangle \\
& =\left(\left(x_{K}, f_{K}\right), x^{*}\right\rangle .
\end{aligned}
$$

In any case $\left(x^{*}\right)^{-1} x^{*} \supseteq \sigma$. Hence the mapping $\bar{x}^{*} \in(S / \sigma)^{*}$ defined by $\left\langle\sigma^{\#} x, \bar{x}^{*}\right\rangle=\left\langle x, x^{*}\right\rangle$ for all $x \in S$ is well-defined, and we shall have $\Phi \bar{x}^{*}=x^{*}$. Thus, in this case, $\Phi$ must be surjective. 
19.

COROLLARY 7. If $S$ is on orthodox band of left R-modules, and $Q$ the greatest inverse homomorphic image of $S$, then $S^{*}$ and $Q^{*}$ are $R$-isomorphic.

20 .

THEOREM 8. Let $S$ be an orthodox band of left $R$-modules and $\tau$ any R-stable congruence on $S$. The mapping $\Psi:(S / \tau)^{\prime} \rightarrow S^{\prime}$, $\bar{x}^{*} \mapsto \Psi\left(\bar{x}^{*}\right)$ defined by $\left\langle x, \Psi \bar{x}^{*}\right\rangle=\left\langle\tau^{\#} x, \bar{x}^{*}\right\rangle$ for any $x \in S$ is an $R$-isomorphism of $(S / \tau)$ ' into $S$ '. Whenever ${ }^{\prime} \subseteq \tau \subseteq \rho, \rho$ being the minimal group congmence on $S$, this mapping $\Psi$ is a surjective $R$-isomorphism of $(S / \tau)^{\prime}$ onto $S^{\prime}$.

Proof. It is clear that the mapping $\Psi$ must be the restriction of mapping $\Phi$ (of Theorem 7) to the maximal submodule $(S / \tau)^{\prime}$ of $(S / \tau)^{*}$; hence $\Psi$ is an $R$-isomorphism of $(S / \tau)^{\prime}$ into $S^{*}$. Since for every $x \in S$, and every $\bar{x}^{*} \in(S / \tau)^{\prime}$, we must have $\left\langle\tau^{\#} x, \bar{x}^{*}\right\rangle \in R$, we conclude that $\Psi \bar{x}^{*} \in S^{\prime}$ for every $\bar{x}^{*} \in(S / \tau)^{\prime}$; thus $\Psi$ is an $R$-isomorphism of $(S / \tau)^{\prime}$ into $S^{\prime}$.

It will be sufficient to show that the mapping $\Psi:(S / \rho)^{\prime} \rightarrow S^{\prime}$, $\bar{x}^{*} \mapsto \Psi \bar{x}^{*}$ defined by $\left\langle x, \Psi \bar{x}^{*}\right\rangle=\left\langle\rho^{\#} x, \bar{x}^{*}\right\rangle$ for every $x \in S$ will be an $R$-isomorphism of $(S / \rho)^{\prime}$ onto $S^{\prime}$. Let $x^{*}$ be any element of $S^{\prime}$. Since $x^{*}$ must be a homomorphism of $S$ into the additive group $R$, we have $\left(x^{*}\right)^{-1} x^{*} \supseteq \rho$. Hence the mapping $\bar{x}^{*} \in(S / \rho)^{\prime}$ defined by $\left\langle\rho^{\#} x, \bar{x}^{*}\right\rangle=\left\langle x, x^{*}\right\rangle$ for every $x \in S$ is well-defined, and we shall have $\Psi_{x^{*}}=x^{*}$. Thus, in this case $\Psi$ must be surjective.

21.

COROLLARY 8. If $S$ is an orthodox band of left R-modules, $Q$ the greatest inverse homomorphic image of $S$, and $G$ the greatest group homomorphic image of $S$, then $S^{\prime}$ and $Q^{\prime}$ are both $R$-isomorphic with right R-module $G^{\prime}$ which is the dual of left R-module $G$. 
22.

THEOREM 9. Let $S$ be an orthodox band of left R-modules, and $S=\bigcup_{K \in Y} S_{K}=\bigcup_{K \in Y} G_{K} \times E_{K}$ its semilattice decomposition. For any $\lambda \in Y$, the moxping $1_{\lambda}^{*}: S \rightarrow R^{\infty}$ defined by $\left\langle x, 1_{\lambda}^{*}\right\rangle=0$ if and only if $x \in \underset{K \geq \lambda}{U} S_{K}$, and $\left\langle x, 1_{\lambda}^{*}\right\rangle=\infty$ otherwise, is an idempotent of $S^{*}$. The maximal submodule $H_{1_{\lambda}^{*}}$ of $S^{*}$ containing $1_{\lambda}^{*}$ is $R$-isomorphic with $\left(\cup_{K \geq \lambda}^{\cup} S_{K}\right)^{\prime}$ and with the right R-module $G_{\lambda}^{\prime}$, which is the dual of the left $R$-module $G_{\lambda}$.

Proof. For any $\lambda \in Y, \underset{K \geq \lambda}{U} S_{K}$ is an $R$-stable subsemigroup of $S$, and $G_{\lambda}$ will be the greatest group homomorphic image of $\underset{\kappa \geq \lambda}{U} S_{\kappa}$. From Corollary 8 it follows that $\left(\underset{K \geq \lambda}{U} S_{K}\right)^{\prime}$ and $G_{\lambda}^{\prime}$ are $R$-isomorphic right $R$-moduies. It is easy to show that $S \backslash\left(\underset{K \geq \lambda}{\mathrm{U}} S_{K}\right)$ is a prime ideal of $S$. From results in the proof of Corollary 5, it then follows that ${ }_{\lambda}^{*}$ must be an idempotent of $S^{*}$. We remark that for any $x^{*} \in S^{*}, x^{*} \in H_{1}{ }_{\lambda}$ if and only if $V_{x^{*}}=\left\{x \in S \|\left\langle x, x^{*}\right\rangle=\infty\right\}=S \backslash\left(\underset{K \geq \lambda}{U} S_{K}\right)$. Hence the mapping $H_{l_{\lambda}^{*}} \rightarrow\left(\underset{K \geq \lambda}{U} S_{K}\right)^{\prime}, x^{*} \mapsto x^{*} \mid \underset{K \geq \lambda}{U} S_{K}$ is an $R$-isomorphism of $H_{l_{\lambda}^{*}}$ onto $\left(U_{K \geq \lambda} S_{K}\right)^{\prime}$.

\section{3.}

COROLLARY 9. We use the same notations as in Theorem 9. Let $Q$ be the greatest inverse semigroup homomorphic image of. $S$ and $Q=\underset{\kappa \in Y}{U} G_{K}$ its semilattice decomposition. For any $\lambda, \mu \in Y, \lambda \geq \mu$, let $\Phi_{\lambda, \mu}$ be the structure homomorphism of $Q$, and ${ }^{t_{\Phi}}{ }_{\lambda, \mu}$ its transpose; then $I_{\mu}^{*} \geq I_{\lambda}^{*}$ in $S^{*} ;$ Let $\Phi_{\mu, \lambda}^{*}: H_{I_{\mu}^{*}} \rightarrow H_{I_{\lambda}^{*}}$ be the structure homomorphism of 
$S^{*}$. For any $\lambda \in Y$ the mapping $\Psi_{\lambda}: H_{1_{\lambda}^{*}} \rightarrow G_{\lambda}^{\prime}, x^{*} \mapsto \Psi_{\lambda} x^{*}$ defined by $\left(\left(x_{K}, e_{K}\right), x^{*}\right\rangle=\left\langle\Phi_{K}, \lambda^{x_{K}}, \Psi_{\lambda^{*}} x^{*}\right.$ for all $\left(x_{K}, e_{K}\right) \in \underset{K \geq \lambda}{\bigcup} S_{K}$ is an R-isomorphism of $H_{1_{\lambda}^{*}}$ onto $G_{\lambda}^{\prime}$, and the following diagram is commutative:

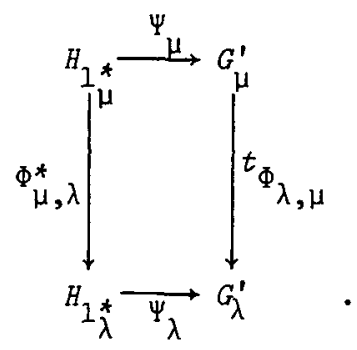

Proof. The mapping $\underset{\kappa \geq \lambda}{U} S_{\kappa} \rightarrow G_{\lambda},\left(x_{\kappa}, e_{K}\right) \mapsto \Phi_{K, \lambda} x_{\kappa} \quad$ is a homomorphism of $\underset{K \geq \lambda}{U} S_{K}$ onto its greatest group homomorphic image $G_{\lambda}$; $\Psi_{\lambda}$ must then be an $R$-isomorphism of $H_{1_{\lambda}^{*}}$ onto $G_{\lambda}^{\prime}$ by Theorem 8 .

Let $x^{*}$ be any element of $H_{1_{\mu}^{*}}$, and $x_{\lambda}$ any element of $G_{\lambda}$. We proceed to show that $\left\langle x_{\lambda}, t_{\Phi, \mu_{\mu} \mu x^{*}}\right\rangle=\left\langle x_{\lambda}, \Psi_{\lambda} \Phi_{\mu, \lambda^{*}} x^{*}\right)$. Indeed,

$$
\begin{aligned}
& \left\langle x_{\lambda},{ }^{t} \Phi_{\lambda, \mu} \Psi_{\mu} x^{*}\right\rangle=\left\langle\Phi_{\lambda, \mu^{x}}{ }_{\lambda}, \Psi_{\mu} x^{*}\right\rangle \\
& =\left\langle x_{\lambda}{ }^{1}{ }_{\mu}, \Psi_{\mu} x^{*}\right\rangle \\
& =\left(\left(x_{K}, e_{K}\right), x^{*}\right) \\
& \text { for all } \kappa \geq \mu, \Phi_{\kappa, \mu} x_{\kappa}=x_{\lambda}{ }_{\mu}, e_{\kappa} \in E_{\kappa}, \\
& =\left(\left(x_{\lambda}, e_{\lambda}\right), x^{*}\right\rangle \text { for all } e_{\lambda} \in E_{\lambda} \text {, } \\
& =\left\langle\left(x_{\lambda}, e_{\dot{\lambda}}\right), x^{*} 1_{\lambda}^{*}\right\rangle \text { for all } e_{\lambda} \in E_{\lambda} \text {, } \\
& =\left\langle\left(x_{\lambda}, e_{\lambda}\right), \Phi_{\mu, \lambda}^{*}\right\rangle \text { for all } e_{\lambda} \in E_{\lambda} \text {, } \\
& =\left\langle x_{\lambda}, \Psi_{\lambda} \Phi_{\mu, \lambda}^{*} x^{* *}\right\rangle
\end{aligned}
$$

We conclude that ${ }^{t_{\Phi}}{ }_{\lambda, \mu} \Psi_{\mu}=\Psi_{\lambda} \Phi_{\mu, \lambda}^{*}$. 
24.

COROLLARY 10. We use the same notations as in Theorem 9 and Corollary 9. Let the structure semilattice of $S$ be a lattice. Consider $V=\bigcup_{K \in Y} G_{K}^{\prime}$, and define multiplication in $V$ by the following: for any $x^{\prime}, y^{\prime} \in V, \quad x^{\prime} \in G_{\lambda}^{\prime}, \quad y^{\prime} \in G_{\mu}^{\prime}$, put $x^{\prime} y^{\prime}=\left(t_{\Phi \vee}{ }_{\lambda \vee \mu, \lambda^{\prime}}\right)\left(t_{\Phi}{ }_{\lambda \vee \mu, \mu} y^{\prime}\right)$. Define the mapping $R \times V \rightarrow V, \quad\left(\alpha, x^{\prime}\right) \mapsto x^{\prime} \alpha$ in the usual way. Then $V$ is a semilattice of right R-modules, and there exists an R-isomompism of $V$ into $S^{*}$. If $Y$ satisfies the minimal condition, $V$ must be R-isomorphic with $S^{*}$.

25.

REMARKS. Corollaries 9 and 10 show that $S^{*}$ could well be named the dual of $S$. If $Y$ is a lattice, the structure semilattice of $V$ is the $v$-semilattice $Y$. The results of [6] make the connections between the structure theorems for $S$ and the structure theorems for $V$ more explicit.

Theorem 7 is quite analogous with a result in [5], $\$ 5$, about the character semigroup of a commutative semigroup, and Theorem 9, Corollary 9, and Corollary 10 are in a certain way analogous with results of [7] and [8] (see also [3], Chapter 5).

The next theorem generalizes the concept of the transpose of an $R$-linear mapping.

\section{6.}

THEOREM 10. Let $S$ and $T$ be orthodox bands of. left R-modules, and $\theta: S \rightarrow T$ an R-Zinear mapping. The mapping $T_{\Theta}: T^{*} \rightarrow S^{*}$, $t^{*} \mapsto T_{\Theta t^{*}}$ defined by $\left\langle x, T_{\Theta t^{*}}\right\rangle=\left\langle\Theta x, t^{*}\right\rangle$ for alz $x \in S$, must be an R-linear mapping of $T^{*}$ into $S^{*}$, and $T_{\Theta\left(T^{*}\right)}$ is embeddable in $\left(S / \theta^{-1} \theta\right) *(\theta S)^{*}$.

Proof. It must be clear that for any $t^{*} \in T^{*}$, we must have $T_{\Theta t^{*} \in S^{*}}$, since $\Theta$ is $R$-linear; it is not difficult to show that 
$T_{\Theta}$ is $R-1$ inear.

Let $t^{*}$ and $v^{*}$ be any elements of $T^{*}$; then $t^{*} \mid \dot{\theta} S$ and $v^{*} \mid \theta S$ are both elements of $(\Theta S)^{*}$ since $\Theta S$ is an $R$-stable subsemigroup of $T$. From the definition of ${ }^{T} \theta$ we have that ${ }^{T} \theta t^{*}={ }^{T} \theta v^{*}$ if and only if

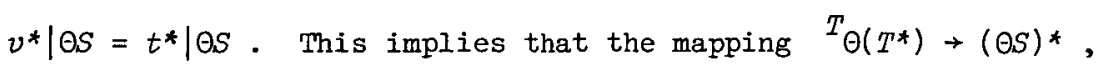

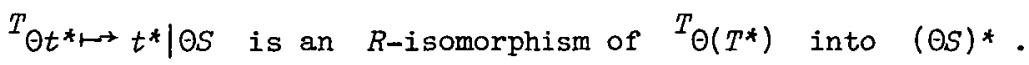

27 .

COROLLARY 11. Let $S, T$, and $\theta$ be as in Theorem 10. The mapping $t_{\Theta}: T^{\prime}+S^{\prime}, t^{* \mapsto}{ }^{t} \theta t^{*}$ defined by $\left\langle x,{ }^{t} \theta t^{*}\right\rangle=\left\langle\Theta x, t^{*}\right)$ for all $x \in S$, must be an R-Zinear mapping of $T^{\prime}$ into $S^{\prime}$, and $t_{\Theta\left(T^{\prime}\right)}$ is embeddable in $\left(S / \Theta^{-1} \Theta\right)^{\prime} \cong(\Theta S)^{\prime}$.

28 .

COROLLARY 12. We use the same notations as in Theorem 10 and Corolzary 11. Let $\rho_{S}$ and $\rho_{T}$ be the minimal group congmences on $S$ and $T$ respectively. Let $\Psi_{S}:\left(S / \rho_{S}\right)^{\prime} \rightarrow S^{\prime}, \bar{x}^{*} \mapsto \Psi_{S^{*}} \bar{x}^{*}$, be the R-isomorphism defined by $\left\langle x, \Psi_{S^{*}} \bar{x}^{*}\right\rangle=\left\langle\rho_{S}^{\#} x, \bar{x}^{*}\right\rangle$ for all $x \in S$, and let $\Psi_{T}:\left(T / \rho_{T}\right)^{\prime} \rightarrow T^{\prime}, \bar{t}^{*} \mapsto \Psi_{T} \bar{t}^{*}$ be defined by $\left(t, \Psi_{T^{*}} \bar{t}^{*}\right\rangle=\left\langle\rho_{T^{*}} t, \bar{t}^{*}\right\rangle$ for all $t \in S$. Then there exists an $R$-linear mapping $\Lambda: S / \rho_{S} \rightarrow T / \rho_{T}$ such that the following diagrams are commutative:

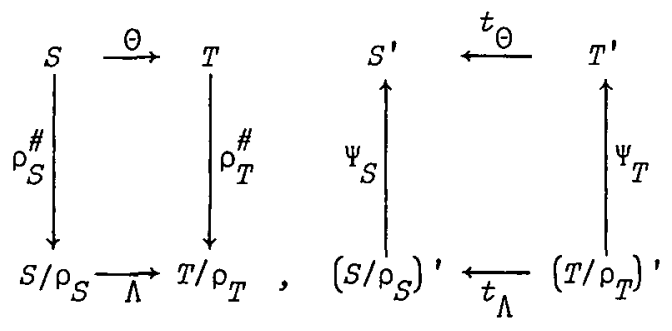

Proof. Since $\rho_{T}^{\#} \Theta$ is an $R$-linear mapping of $S$ into the left 
$R$-module $T / \rho_{T},\left(\rho_{T}^{\#} \theta\right)^{-1}\left(\rho_{T}^{\#} \Theta\right)$ must be an R-stable group congruence on $S$, and, since $\rho_{S}$ is the minimal group congruence on $S$, we must have $\rho_{S} \subseteq\left(\rho_{T}^{\#}\right)^{-1}\left(\rho_{T}^{\#}\right)$; this implies that $\Lambda$ is a well-defined $R$-linear mapping of $S / \rho_{S}$ into $T / \rho_{T} \cdot{ }^{t} \Lambda$ is then an R-linear mapping of $\left(T / \rho_{T}\right)^{\prime}$ into $\left(S / \rho_{S}\right)^{\prime}$ which is defined by $\left\langle\rho_{S}^{\#} x,{ }^{t} \bar{t}^{*}\right\rangle=\left\langle\Lambda \rho_{S}^{\#} x, \bar{t}^{*}\right\rangle$ for all $x \in S$ and all $\bar{t}^{*} \in\left(T / \rho_{T}\right)^{\prime}$; but since $\Lambda \rho_{S}^{\#}=\rho_{T}^{\#} \Theta$, we then have

$$
\begin{aligned}
\left\langle\rho_{S}^{\#} x,{ }^{t} \bar{t}^{*}\right\rangle & =\left\langle\rho_{T}^{\#} \Theta x, \bar{t}^{*}\right\rangle \\
& =\left\langle\Theta x, \Psi_{T} \bar{t}^{*}\right\rangle \\
& =\left\langle x,\left({ }^{t} \Psi_{T}\right) \bar{t}^{*}\right\rangle \\
& =\left\langle\rho_{S^{*}} x,\left(\begin{array}{ll}
\Psi_{S}^{-1} & \left.\left.t_{\Theta \Psi_{T}}\right) \bar{t}^{*}\right\rangle
\end{array}\right.\right.
\end{aligned}
$$

for all $x \in S$ and all $\bar{t}^{*} \in\left(T / \rho_{T}\right)^{\prime}$; hence ${ }^{t} \Lambda=\Psi_{S}^{-l} t_{\Theta \Psi}$.

\section{References}

[1] N. Bourbaki, Éléments de mathématique, Fascicule VI. Première partie, Livre II, Algèbre; Chapitre 2, Algèbre Zinéaire, $3 \mathrm{e}$ édition (Hermann, Paris, 1967).

[2] A.H. Clifford, "The structure of orthodox unions of groups", Semigroup Forum 3 (1971/1972), 283-337.

[3] A.H. Clifford and G.B. Preston, The algebraic theory of semigroups, Volume I (Mathematical Surveys, 7. Amer. Math. Soc., Providence, Rhode Island, 1961).

[4] *J.M. Firsov, "Everywhere defined semimodules" (Russian), Sumaries of talks of the all-union algebraic symposium, 360-361 (Gomel, 1975).

[5] E. Hewitt and H.S. Zuckerman, "Finite dimensional convolution algebras", Acta Math. 93 (1955), 67-119. 
[6] F. Pastijn and H. Reynaerts, "Semilattices of modules", submitted.

[7] *Stefan Schwarz, "The theory of characters of finite commutative semigroups" (Russian), Crechoslovak Math. J. 4 (79) (1954), 219-247.

[8] R.J. Warne and L.K. Williams, "Characters on inverse semigroups", Caechoslovak Math. J. 11 (86) (1961), 150-155.

[9] F. Wuytack and J. Depunt, "Operators over I-collections of modules", Bulz. Soc. Math. Belg. 17 (1965), 37-54.

[10] Miyuki Yamada, "Strictly inversive semigroups", Bulz. Shimane Univ. Natur. Sci. 13 (1963), 128-138.

[11] Miyuki Yamada and Naoki Kimura, "Note on idempotent semigroups II", Proc. Japan Acad. 34 (1958), 110-112.

* The author has not had access to [4] and [7], which are quoted at second hand. Editor.

Dienst Hogere Meetkunde,

Rijksuniversiteit Gent,

KrijgsIaan,

Gent,

Belgium. 